\title{
Intelligent Management of Multiple Access Schemes in Wireless Body Area Network
}

\author{
Jing Zhou ${ }^{\mathrm{a}, \mathrm{b}}$, Aihuang Guo ${ }^{\mathrm{b}}$, Hung T. Nguyen ${ }^{\mathrm{a}}$ and Steven $\mathrm{Su}^{\mathrm{a}}$ \\ ${ }^{a}$ Faculty of Engineering and Information, University of Technology, Sydney, Australia \\ ${ }^{\mathrm{b}}$ Department of Information and Telecommunication, Tongji University, China
}

E-mail: Jing.Zhou-2@student.uts.edu.au, tjgah@tongji.edu.cn, Hung.Nguyen@uts.edu.au, Steven.Su@uts.edu.au

\begin{abstract}
Wireless Body Area Network is a novel technology to provide efficient, convenient and safe health, medical and personal entertainment services but it still faces longstanding challenges in the application. A smart communication standard is essential to solve some problems in wireless data transmission and optimize system performance. This paper proposes an intelligent management of multiple access schemes for contention access and contention free access with game theory. The strategies are determined by requirements of applications and channel status. Nodes in a network choose various strategies for best payoff according to different conditions including normal status, packets-oriented services, emergent situation and bad link states with specific utility functions. The novel management model supports flexible resource allocation and enhances the system performance, which are evaluated by simulations of Castalia under various conditions.
\end{abstract}

Index Terms - Wireless Body Area Network, Medium Access Control, Game Theory

\section{INTRODUCTION}

\section{A. Background of Wireless Body Area Network}

Healthcare is a critical issue in the current world due to the rapid growth of demands in health and medical related services including baby care, diet monitor, sports assists, diseases, people caring and monitoring[1, 2]. Simultaneously with the growing population of old people, new technologies and applications are required to provide auxiliary methods for elderly independence and reduce healthcare costing [3]. Medical organizations and institutions always make effort to generate efficient, convenient and safe services for people who are in need at any time and any places. But because of the limited financial support, insufficient devices and professional human resources it is not easy to solve some problems and guarantee reliable services with traditional methods. Wireless body area network (WBAN) is a new technique to challenge the general model and bring benefits in health and medical applications [4-6].

WBAN uses sensors on, around or inside the human body to collect biological and environment parameters for

Manuscript received November, 2014; revised February, 2015; accepted February, 2015.

corresponding author: Jing Zhou, Jing.Zhou-2@student.uts.edu.au further analysis or provide monitoring. Compared to traditional wireless sensor networks the human body environment and QoS in medical services bring new challenges in WBAN application, such as extreme energy efficiency, unique characteristics of the wireless channel, low latency and high data integrity $[7,8]$. Furthermore the requirement for reliability of WBAN is higher than classical WSN due to the sensitive data, health care and medical services. WBAN is expected to avoid failure and . enhance throughput for various applications. Therefore new health communication standards and approaches must be developed to meet stringent technical requirements. Therefore it requires new approaches and appropriate protocols to improve the reliability and support various functions in health and medical related scenarios. Medium access control is of paramount importance because it arranges how the spectrum is shared across users and flows which directly affect the system throughput, reliability, quality of service and fairness. Therefore MAC protocol needs to be designed to suit specific needs of specialized applications.

There are several wireless local area network protocols such as Bluetooth, Zigbee and WiFi for short distance services but they are not available for WBAN, which is specially targeting for health and medical application because specific working conditions are not considered in these standards. IEEE 802.15.6 Task Group (TG) developed an optimized communication standard to serve a variety of applications including medical, health and personal entertainment [9]. It outlines the basic elements including packet formats and message exchange protocols in medium access control (MAC) and physical layers to support a reliable wireless communication. But it still leaves open questions for further development, such as: how to manage the various access schemes including contention- based, scheduled, or improvised access? Is it available to increase the resource utilization and performance with intelligent control [7]? So there are still a lot of work to be done and the medium access control is a key factor [10], which has been studied by many researchers from different aspects.

\section{B. Related works}

Recently a wealth research of MAC has been done for enhancing the performance of WBAN. Sana Ullah derived numerical formulas to determine the maximum 
throughput and minimum delay of IEEE 802.15.6-based CSMA/CA Protocol [11]. They prove and evaluate the throughput and delay bounds for different data rates and frequency bands with one sender and one receiver. Shih Kuei-Ping proposed a virtual carrier sense MAC protocol with power controlling to avoid collisions [12]. It effectively reduces the energy consumption by a collection of stations and prevents collisions due to Point issue. Pangun Park designed an adaptive MAC protocol in control and monitoring applications [13]. A model is proposed based on Markov chain to minimize the power consumption and guarantee delay limitation and reliability. A. M. Asadi discussed a framework and surveyed proposed algorithms as opportunistic scheduling. In the application a user attempts to maximize the network throughput to meet certain constraints [14] . Mario Eagalj used a game-theoretic scheme to analyze the selfish behavior of nodes in CSMA/CA for a simple, localized and distributed protocol which successfully supervises multiple selfish nodes to reach a Pareto-optimal Nash equilibrium[15]. Boulis studied the trade-offs caused by a mix of two MAC techniques: contention-based access, and pollingbased access. This paper also presents some MAC techniques including dynamic allocation of slots, retransmissions and power control based on studies of the BAN channel [16]. Tian Jun proposed a Game-theory Model based on CSMA Protocol in Wireless Network for more throughputs[17]. The analytical can get the transmit power and optimal carrier sensing threshold with maximizing the total network throughput. These research concerned TDMA or CSMA/CA respectively but did not consider multiple access schemes together in the application.

Usually contention-free access and contention access coded are simultaneously deployed in the application. The scheduling problem might be quite hard under various channel conditions and error-prone wireless channels. Therefore taking into account the requirement of applications and channel status, an intelligent control strategy is necessary for flexible and ideal resource allocation and better performance. So we establish an optimal strategy to manage various access schemes under different situations including services and link states. To the best of our knowledge it is the first time to propose a comprehensive management model of different access schemes for WBAN. The proposed scheme can be applied to generate an efficient and smart MAC protocol and the performance is evaluated by a set of simulations.

The contributions contains: 1) We define a series of utility functions with considering various situations in data transmission. These specific definitions can facilitate network to adapt to communication requirement and channel states. 2) An intelligent medium access scheme is proposed to obtain flexible and efficient allocation of resource and bring benefits for WBAN in different applications.

The rest of the paper is organized as follows: Section II introduces the main access mechanisms and their features respectively. Section III analyses the WBAN performance including throughput, latency and energy consumption under different mechanisms. Section IV proposes the intelligent management model. Section V illustrates simulations and results. Section VI makes conclusions.

\section{OVERVIEW OF MEDIUM ACCESS MECHANISMS}

The MAC protocol of WBAN aims to manage the usage of the medium access mechanism for a short range, low cost, low power and highly reliable wireless communication. Generally there are three main medium access mechanisms for WBAN including: contention access of CSMA/CA, scheduled access of guaranteed time slots and improvised access of polling [9].

\section{A. CSMA/CA}

CSMA/CA is a connect-less service to transfer asynchronous message without guarantee of bandwidth and latency $[11,18]$. It adapts quite well in the variable conditions of traffic and is quite robust against interferences. In the CSMA/CA scheme a node begins with listening the status of channel before sending. If the channel is found to be idle, the node sends the first packet in the transmit queue. If the channel is busy (for example another node is in transmission or interference), the node waits the end of the current transmission and a random period of time, which means starting the contention. The CSMA/CA applies collision avoidance instead of collision detection in wired Ethernet because it is difficult to detect collision in wireless communication. But it can't acquire high efficiency for connection oriented services.

\section{B. Guaranteed time slots}

Guaranteed time slots is a time division multiplex access (TDMA) scheme[19]. The base station or the coordinator is responsible for regulation of nodes in a network. The channel is divided into time slots, which are normally of fixed size. Every node in the network can be allocated one or more slots where it can transmit data. Slots are usually constituted in a frame and the coordinator determines the frame structure in a beacon. Each node follows the instruction in the beacon. This mechanism is suitable for predictable needs and is available to achieve low latency and guarantee bandwidth. Due to the inflexibility and connection oriented it is not appropriate for variable size packets and the burst traffic. This mechanism heavily depends on the quality of the channel.

\section{Polling}

In polling access the coordinator totally controls over the channel, but the frame structure is no more fixed and variable size packets are allowed to transmit. With a specific poll packet, the coordinator triggers the transmission scheme by the node. When the node receives a poll packet, and then sends what it wants to transmit.

Polling is a scheme between a connection oriented service (TDMA but with flexible packet size) and 
connection less-service (asynchronous transmission). The coordinator can either totally use polling of the network to check if all nodes have something to send ,which is available for limited nodes, or use reservation slots where each node can request a connection to transmit data.

\section{IEEE 802.15.6 Communication Modes}

The IEEE 802.15.6 standard defines a MAC layer that provides services for Physical layers including Narrowband, Ultra-wideband and Human Body Communications (HBC) layers[20]. According to the standard the WBAN is consisted of one hub/coordinator and some nodes (sensors), ranging from zero to maximum WBAN Size. A single coordinator or hub controls the entire operation in data transmission. The time axis or channel is usually divided into beacon periods or superframes of equal length. Each superframe contains a number of allocation slots and these slots have equal time duration. The coordinator transmits beacons to determine the superframe boundaries and allocate slots.

The MAC frame structure contains three parts: Beacon, Downlink and Uplink. Beacon is used to describe frame structure and guarantee synchronization. It also contains information which should be periodically broadcasted to nodes. The Downlink is set for data transmission from the coordinator to nodes and the function of Uplink part is vice versa. The Uplink part usually has two sub-parts: Contention Access Period (CAP) and Contention Free Period (CFP). CAP is based on CSMA/CA or slotted ALOHA and it is also used to transmit MAC control information. In CFP Guaranteed Time Slot (GTS) is designated to nodes for scheduled data transmission.

This standard supports several communication modes:

- Beacon Mode with Superframe Boundaries. In this Mode, the hub transmits beacons in active superframes. The active superframes may be followed by several inactive superframes if there is no scheduled transmission. The superframe structure is divided into Exclusive Access Phases (EAP), Random Access Phases (RAP), a Managed Access Phase (MAP), and a Contention Access Phase (CAP).

- Nonbeacon Mode with Superframe Boundaries: In this mode, the hub operates during the MAP period only.

- Nonbeacon Mode without Superframe Boundaries: In this mode, the hub provides unscheduled polled or posted allocations or a combination of both.

Usually the beacon mode with Superframe Boundaries is widely accepted in application so this model is applied in our study. Although it defines several access mechanisms, such as random, improvised, unscheduled and scheduled access, it doesn't explicitly illustrate how to apply these mechanisms and obtain the efficient usage of medium. The duration of different schemes is not adaptively allocated based on the traffic characteristics.

\section{PERFORMANCE ANALYSES}

The purpose of WBAN is to offer reliable, flexible, high channel efficiency and cost saving services for professionals and patients. In order to evaluate the performance of a WBAN, latency, energy consumption, successfully received packets and breakdown packets are key factors to be measured with simulation.

\section{A. Latency}

In a network, latency is an expression of how much time it takes for a packet of data from one designated point to another. For transmitting critical data in WBAN time constraint is extremely critical so that data should be received within minimum latency. Usually latency can be given by (1):

$$
\text { Latency }=O_{\text {waiting }}+T x_{\text {Time }}=O_{\text {waiting }}+\frac{F}{R}
$$

Where $\mathrm{O}_{\text {waiting }}$ is the overall waiting time, which contains queuing latency, acknowledgement time and contention window period in CSMA/CA. $\mathrm{Tx}_{\text {Time }}$ is the transfer time for data, which is determined by $\mathrm{F}$ and $\mathrm{R}, \mathrm{F}$ is the data frame size and $\mathrm{R}$ is data bit rate.

For example: as defined in [21] the total duration of the frame:

$$
T_{X \text { Time }}=8 * \frac{L_{P H Y}+L_{M A C H D R}+L_{a d d r e s s}+x+L_{M A C F I R}}{\text { Rdata }}
$$

LPHY is the Length of the PHY header with 6 bytes, LMACHDR is the Length of the MAC header with 3 bytes, LADDRESS is the Length of the MAC address info field and the length of 1 PAN-identifier is 2 bytes, $x$ is the number of bytes that are received from the upper layer, LMACFTR is the Length of the MAC footer with 2 bytes and Rdata is the Raw data rate.

Therefore latency increases linearly with the amount of nodes and it is terrible for emergency data if they have to wait a long queuing. If emergency data use reserved slots and are transferred without queuing latency then the system can fulfill transmission in shortest period.

\section{B. Energy consumption}

The energy consumption is different from various communication techniques. For example energy is consumed for sensing operation. A large amount of energy is wasted in "idle listening" period. When two nodes want to communicate with each other, both of them should be active during this time. If there is not proper scheduling, the receiving node does not know the purpose of the transmitting node. The receiving node has to continuously listen to the channel.

The transmission power is set by a transmitter and the energy in our system is the model in reference [22] and can be defined by (2):

$$
E(R)=P_{l} t_{l}+P_{t x} \times\left(t_{\text {tone }}+\frac{F}{R}\right)+P_{r x}\left(t_{\text {tone }}+\frac{F}{R}\right)
$$


where $P_{l}$ is consumed power in the state of listening, $\mathrm{t}_{l}$ is time of listening, $\mathrm{t}_{l}, \mathrm{t}$ tone are channel listening and tone sending time respectively, $\mathrm{P}_{\mathrm{tx}}$ is consumed power in the state of transmitting, $\mathrm{P}_{\mathrm{rX}}$ is the consumed power consumption in the state of receiving state. So it is available to obtain the network's energy expenditure at various rates.

\section{Throughput}

The throughput is related to the data rate and the probability of successful packets during the transmission. It represents the real successful data in specific period and can be defined as the average amount of packets which are transmitted in a unit time.

For CSMA/CA the throughput can be defined as the ratio of the payload size to the total deliver time for each payload [11] in (3):

$$
S_{C}=\frac{B^{*} L}{\operatorname{Deliverytime}(x)}
$$

Where L is the payload bytes or slots, which depends on the definition in the application, $\mathrm{B}$ is the bits in one packet payload, Deliverytime $(x)$ is associated with average contention windows, data transmission time, immediate acknowledgement time, short inter frame spacing time and propagation delay.

While for GTS the throughput can be defined as (5) [19]:

$$
S_{G}=\frac{P_{S}(t) L}{\theta T_{s S}}
$$

$P_{S}(t)$ is the probability of a successful GTA allocation, $L$ is the length of payload, $\theta$ is the number of superframe slots, $T_{S S}$ is a superframe slot length.

For a whole network when CSMA and GTS are both used in the MAC, the total throughput can be defined in (6):

$$
S=\frac{S_{C} \times N_{C}+S_{G} \times N_{G}}{N_{C}+N_{G}}
$$

where $\mathrm{N}_{\mathrm{C}}$ is the number of slots for CSMA/CA, which is the period for RAP in 802.15.6. $N_{G}$ is the number of slots for GTS. So the normalized system throughput is associated to the access schemes, which affects the successful probability, the effective slots and latency for data transmission.

\section{Successful received packets}

Usually the system throughput of the superframe is decided by the successfully payload in the delivery time. But sometimes it is not easy to accurately measure or calculate the time and the throughput is not the primary factor in special occasions so successful received packets in (6) can also be used to evaluate the performance to ensure the maximum packets are received:

$$
S_{i}=P_{S i} \times L \times A
$$

where A is a normalization constant to convert payload to packets in designated period, $\mathrm{L}$ is the payload of bytes or slots. $P_{s i}$ is the probability that any node successfully transmit data in designated period. The probability can be affected by different access schemes and channel states.

\section{INTELLIGENT MEDIUM ACCESS MODEL AND SIMULATIONS}

As previously described, either GTS or Polling usually requires a service slot or reservation slot. If the MAC is connection oriented and the rate of new connection is low, maybe a single service slot is enough. If the MAC is packet oriented and the requested rate is high, then the protocol needs to offer more reservation slots. On the other side for some variable data rate, CSMA/CA is the preferred scheme due to the flexibility. Usually static allocation of resource cannot obtain the best performance under variable conditions and environment. Therefore intelligent management of various access schemes is in need and can effectively improve the utilization of bandwidth and obtain better performance. All nodes in a network act like competitors and they share the same channel to transmit data. Each node's action will affect other nodes' access. This competition is consistent with the principle of Game theory. So Game theory can be used as an effective and smart method to implement the resource allocation in MAC for WABN. Hence we propose an intelligent manage access mechanisms with game theory for various conditions including traffic requirements and channel states. The precondition of the WBAN is a star topology and all the nodes are competitive to send individual data packets.

So each node's channel access will directly affect other nodes in wireless channel. With different utility functions to represent all types of demands the Nashequilibrium is analyzed to allocate resources optimally.

\section{A. Introduction of Game theory}

Game theory is a mathematic method to study the interactions between several decision-makers who have conflicts or common interest. A game model comprises a set of players, which choose their actions in each period of the game to maximize that period's expected payoff. Payoff usually represents profit, quantity, utility, or other continuous measurement or simply illustrates the desirable outcomes[23]. Game theory has been used for medium access control. For example: Tao cui provided a general game theoretic framework for contention based medium access control [24]; Li jun use A game-theoretic approach to control contention [24, 25].

A static non-cooperative game model is used for access control in this study. The non-cooperation game reflects a competitive situation where every player needs to take its decision independently of other players, giving the possible choices of other players and their effect on the players' utilities. 
Definition 1: A general random access game $G$ is defined as a triple $\mathrm{G}=\left\{\mathrm{N},\left(\mathrm{S}_{\mathrm{i}}, \mathrm{i} \in \mathrm{N}\right),\left(\mathrm{U}_{\mathrm{i}}, \mathrm{i} \in \mathrm{N}\right)\right\}$, where $N=(\mathbf{1}, \ldots, n)$ is the finite set of players, $\mathrm{S}_{\mathrm{i}}$ is the available strategies and $\mathrm{U}_{\mathrm{i}}$ is the utility function to evaluate the payoff. In our analysis $\mathrm{N}$ represents wireless nodes in a WBAN. Choosing the access scheme is the strategy that every node uses. $\mathrm{U}_{\mathrm{i}}$ is determined by the defined function for various scenarios.

In game theory, Nash Equilibrium (NE) is a solution concept of a game involving two or more players, in which each player is assumed to know the equilibrium strategies of the other players, and no player has anything to gain by changing only its own strategy unilaterally [8]. We use the best response concept to acquire the Nash Equilibrium. If a player A has a dominant strategy $S_{A}$, then there exists a Nash equilibrium where A plays $S_{A}$. For two players $\mathrm{A}$ and $\mathrm{B}$, there exists a Nash equilibrium in which A plays $S_{A}$ and B plays a best response to $S_{A}$. If both $\mathrm{A}$ and $\mathrm{B}$ have strictly dominant strategies, there exists a unique Nash equilibrium in which each plays his strictly dominant strategy.

The performance of a network can be analyzed by various utility functions for MAC. A utility function generally describes a given player's preferences for all possible results and a higher value of result means that the preference is better. These specific definitions are useful for different conditions and strategies to approach the equilibrium of the game, which is a stable state that nodes choose the best action for a given utility. Therefore a series of utility functions is proposed for various conditions.

We formulize the system model into a static game theory model, where all players make decisions rationally either simultaneously or asynchronously without knowing the decisions of other players. We design the utility function to analyze the behavior of sensors and acquire the best response.

\section{B.Normal status}

For normal network status we use game theory to obtain the maximum payoff with defined utility function. Nodes (sensors) are players in this game and all nodes make their decision simultaneously. Since we assume that a player's object is to maximize the utility and the coordinator determines strategies to acquire the maximum payoff in normal status.

Because packets, latency and energy are key parameters to evaluate the system performance we use these factors to define the utility of balanced performance in (8) to represent the payoff,

$$
U_{N}=\sum_{i=1}^{n} U(i)=\sum \frac{U\left(S_{i}\right)}{K \times U\left(L_{i}\right) \times U\left(E_{i}\right)}
$$

where $\mathrm{U}(\mathrm{S})$ is the successful received packets in designated period, $U(L)$ is the average latency for packets, $\mathrm{U}(\mathrm{E})$ is the consumed energy by all nodes and $\mathrm{K}$ is a coefficient to adjust the utility according to network requirement and conditions. When the utility is energy- oriented, the $\mathrm{K}$ is set as the maximum value to emphasize the influence of consumed energy. The constraint for the function is that the total allocated slots in the control process cannot exceed maximum available slots $B$. The utility goes up with the increasing successful packets and the decreasing latency and consumed energy. By varying the access scheme the system gets the maximum $U_{N}$ as the best response as the equilibrium.

\section{Packets oriented}

For some packets oriented services the maximum amount of successful packets is a major demanded factor, so nodes should be allocated enough slots to guarantee the requirement. For example if the data rate is $1024 \mathrm{Kbps}$ and the slot allocation length is chosen to be $10 \mathrm{~ms}$. A 128 byte data packet needs $1 \mathrm{~ms}$ to be transmitted with the BAN radio so the total period for a packet (radio transition times $+\mathrm{TX}+\mathrm{ACK}$ ) is $1.16 \mathrm{~ms}$. It means each allocation slot can hold 8 packets. Considering the packet rate the required slots are calculated by (9):

$$
\text { Slots }=\frac{P R^{*} B P}{N P}
$$

$\mathrm{PR}$ is the packets rate, $\mathrm{BP}$ is the time of a beacon period and NP is the number of packets in a slot. Therefore the utility for packets oriented services is decided by (10):

$$
U_{P}=\sum_{i=1}^{n} U\left(S_{i}\right)
$$

$S_{i}$ is the slots required by each node. For a node of highest rate 100 packets/second, calculating a theoretical required number of slots is 4 . At this condition the node gets the highest successful packets and the system obtains the best value of the successful received packets from all nodes as shown in figure based on the simulation. When the allocated slots are 4 the amount of successful received packets reaches the maximum and more slots can't improve the performance. Also for rate of 50 packets/second, two scheduled slots are enough for data transmission. So choosing suitable slots in GTS can get best payoff of the utility and save the time for transmission.

\section{Emergency}

For WBAN application if the coordinator detects emergency information, such as: a person's falling or abnormal signals of critical specific nodes by data analysis[26], it turns into emergency state and triggers new allocation at the next frame. At this stage, some nodes may increase their sampling rate to guarantee reliable monitoring and the polling mechanism is used for high data flow without considering energy consumption.

As defined in 802.15.6, if the coordinator gets a packet of one node whose more Data field is 1 , it means this node has one extra packet to transmit. After the coordinator counts the number of such packets, it sends a poll message enclosed in the ACK packets. Therefore nodes obtain required polling-based slots.

These nodes are treated as selfish players[15], which try their best to occupy the time of channel for maximum transmission probability. 
At this condition the successful packets (11) of specific nodes or one node is used to define the utility function.

$$
U=\sum_{j=k}^{m} U\left(P_{j}\right), \quad k, m \in(1,2, \ldots n)
$$

where " $\mathrm{k}, \mathrm{m}$ " is the serial number of specific nodes.

These selfish players can increase their expected payoff at the expense of other nodes by using polling strategy. So we focus on the individual utility of each sensor in this condition.

\section{E. Bad link status}

The link status comes from the recently information of the channel state. If the amount of consecutive breakdown packets increases heavily and then reaches a threshold, it means the channel condition is worse. The access scheme should be modified and the increasing of random access period can decrease the possibility of breakdown packets. Because GTS is highly depends on the channel status while CSMA/CA is flexible in slots using. So using more slots of CSMA/CA is preferred for this condition and the throughput in (6) is used as the utility. The allocation of large random access slots can bring benefits to the value of the payoff for this condition.

\section{SIMULATION AND ANALYSIS}

We consider a body area system with $\mathrm{N}$ wireless sensors to one designated receiver (coordinator) and we assume all nodes are working in the same communication range (i.e., every node can hear other nodes to avoid complicated problems caused by the hidden terminal). The simulation is executed in Castalia 3.2 based on the protocol of IEEE 802.15.6 because it already defines the MAC frame format and communication modes. Some simulation parameters are presented in table 1.

TABLE I. SIMULATION PARAMETERS

\begin{tabular}{|l|l|}
\hline Parameters & Values \\
\hline Physical Data Rate & $1024 \mathrm{kbps}$ \\
\hline Network topology & Star \\
\hline Packet size & 128 bytes \\
\hline Simulation time & 50 seconds \\
\hline Start up Delay & 1 second \\
\hline MAC Buffer Size & 48 bytes \\
\hline Tx Output Power & $-10 \mathrm{dBm}$ \\
\hline Receiver Sensitivity & $-87 \mathrm{dBm}$ \\
\hline Time slot size & $10 \mathrm{~ms}$ \\
\hline
\end{tabular}

The duration for each simulation operation is 50 seconds and the results are averaged measurement over 5 repeated runs.

Simulation 1: For normal status, we set simulation for 6 nodes (one coordinator and five nodes for data acquisition) in a wireless body area network, in which the data rates of sending nodes are 25 packets/second, 50 packets/second and 100 packets/second respectively.

The utility is calculated to evaluate the access scheme as shown in Fig 1. The y axis is the utility and the $\mathrm{x}$ axis represents different strategies. "No-polling\&no
GTS" means only CSMA is deployed in the MAC, "noplooing\&GTS” means CSMA and GTS are deployed in the MAC, "polling\&GTS" means polling and GTS are both used in the MAC. For low data rate (25 packets/second with blue bar), three different strategies get the similar results. While for high data rate (100 packets/second with pink bar) polling\&GTS obtain the maxium payoff. At the same situation CSMA (nopolling\&noGTS) the payoff of Utility is the lowest. Therefore we can use the function, which is defined by successful received packets, latency and consumed energy, to get a balanced evaluation and choose the appropriate mechanism for all nodes to make a more efficient use of the wireless medium.

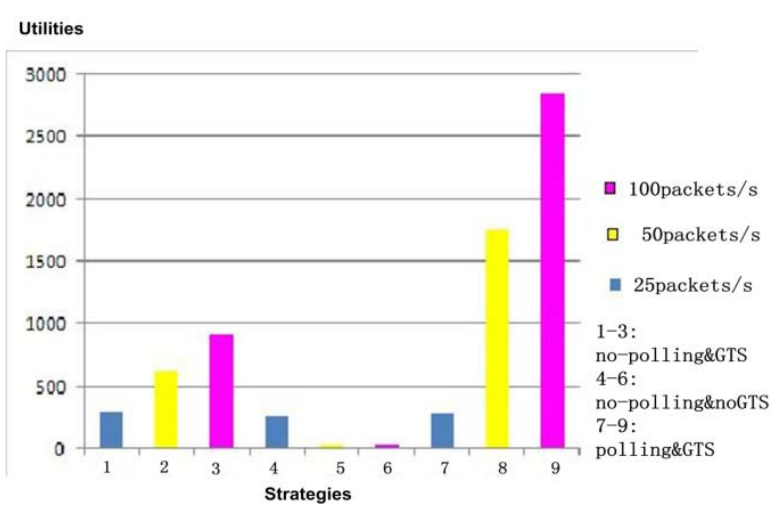

Figure 1 Utilities of various access schemes

For normal wireless protocol of CSMA/CA, when a device wants to transmit data, the device waits for a random period of back off before trying to access the channel. The back off time is randomly generated in the interval [0 , 2BE-1], This will cause more collisions and then reduce the network throughput, increase latency especially for high data rate. With intelligent manage the access, the resource can be dynamically allocated according to rate and acquire better utility as shown in Fig. 2.

Simulation 2: For packets-oriented condition we acquire the payoff of all successful packets with different allocated slots and the result is shown in Fig. 2. The y axis is the average packets received from all nodes and the $\mathrm{x}$ axis is the sending rate for each node measured in packets/second. Nodes are sending packets with 50 packets/second, 100 packets/second and 200 packets/second.

According to (6), adequate slots assignment is the primary factor to ensure the best response in the game. For high data rate of 200 packets/second in this simulation 6 slots is the best response for 5 nodes in competition. When data rate is low the requested GTS is small. Otherwise nodes demand more slots for maximum payoff. From Fig. 2 we can see when the rate is 50 packets/second, 2 slots and 6 slots in GTS access can obtain the same result. When the rate is 100 packets/second and the access scheme of 4 slots is allocated in GTS the coordinator can receive the most packets from every node. While the rate increases to 200 
packets/second, the overall received packets do not increase and keep the same value as shown in the green line, which means the slots allocation can't meet the requirement for high data rate. So when the rate is 200 packets/second 6 slots for GTS is the best strategy because it acquires more packets. Therefore dynamical adjust slots allocation according to operation requirement.

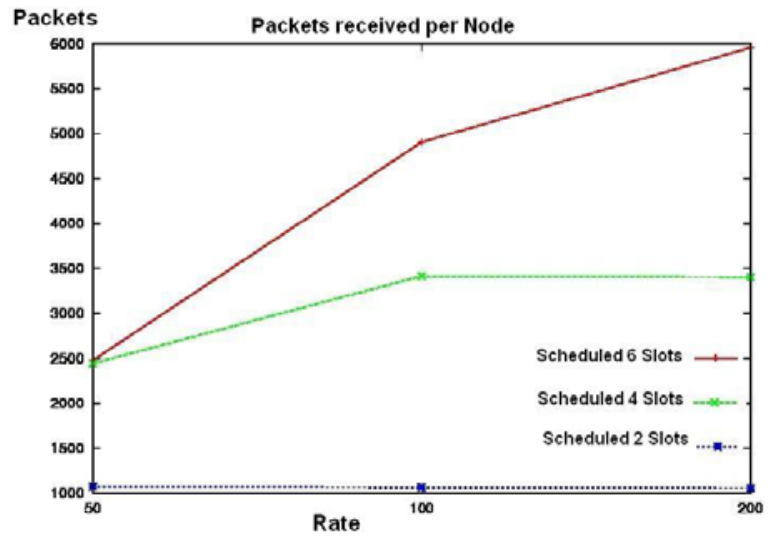

Figure 2 Packets-oriented Access

Simulation 3: For emergency situation we assume a network, in which a node requires urgent services if abnormal signals are detected. Then the scheme of polling is triggered and specific slots are allocated for it. This node is regarded as a selfish player to maximize its payoff without considering other nodes' utility. For this situation, we use (11) to get the best response of node 2 with choosing specific slots. In Fig. 3 we notice that in this situation the ratio of breakdown packets is the smallest when the node 2 uses the polling scheme. It means the ratio of successful packets of this node, which is shown in pink color, is the highest in all nodes. At this situation this player (node 2) obtains its best utility with smart decision due to the requirement.

Simulation 4: For bad link status we use a channel with high interference to test the access scheme. The period of contention access of 2 slots, 6 slots and 16 slots is defined as short CAP, medium CAP and long CAP respectively in figure 4. These strategies are outlined as blue, red and green bar. As defined before, the utility is determined by throughput to guarantee the quality. For low data rate (25 packets/second) the increase of contention access period does not improve while for high data rate (100 packets/ second) the raising of utility is obvious. Therefore for high data rate in bad link status using long contention period is beneficial to the system performance because guaranteed time slots is fixed allocation cannot adjust to variable channel quality.

From the above analysis, it is clearly to see that due to the complicated situations in WABN an intelligent management of MAC is necessary to adapt to various requirement. Effect utility functions are vital to evaluate the payoff and change strategies.

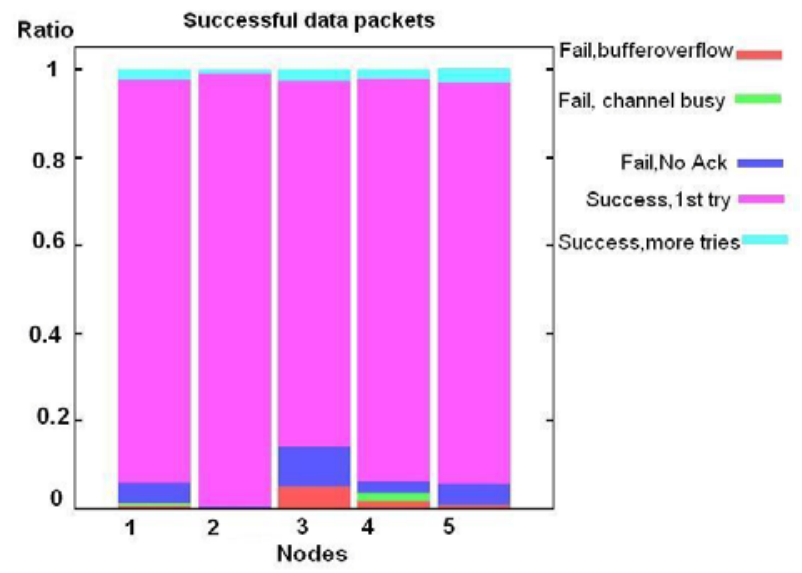

Figure 3 Emergency service

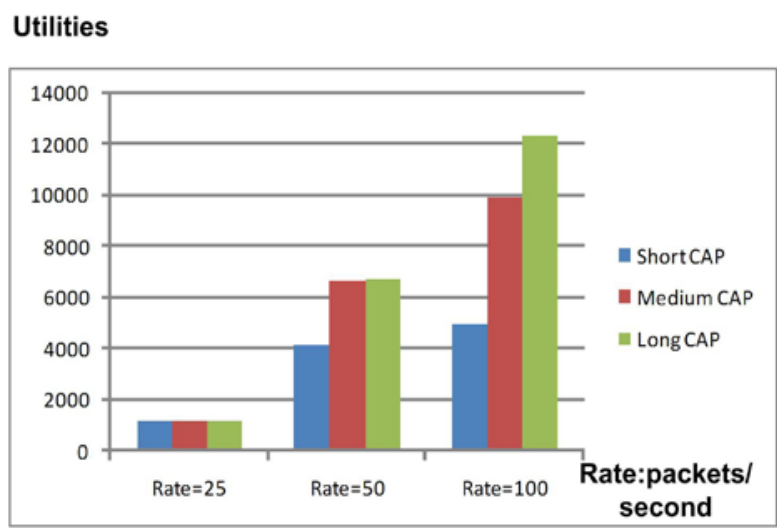

Figure 4 Bad link statuses

\section{CONCLUSIONS}

For WBAN medium access control in wireless communication is a key factor to improve the system performance. MAC protocols should be designed to accommodate changes in network conditions and traffic characteristics. Using game theory to intelligently manage multiple access schemes is proposed in this paper for better performance. Players in the game model choose various strategies in different situations to maximize the expected payoff. The coordinator is responsible for arrange access schemes to obtain better resource allocation or meet specific requirement. All nodes observe the demand and channel state to ensure the dynamic and intelligent model to be implemented. Therefore, the final compromise solution is to achieve the optimal result with multiple constraints.

The proposed model is evaluated in the simulation to illustrate the benefits. In the future research cognitive knowledge, Contextual information and comprehensive conditions in the network will be considered to ensure high quality of services.

\section{REFERENCE}

[1] Y. Zhang, L. Yu, and L. Wang, "High Access Performance Strategy for Next Generation 
Healthcare Networks," Journal of Networks, vol. 9, pp. 1477-1484, 2014.

[2] J. Penders, B. Gyselinckx, R. Vullers, M. De Nil, V. Nimmala, J. van de Molengraft, F. Yazicioglu, T. Torfs, V. Leonov, P. Merken, and C. Van Hoof, "Human++: From technology to emerging health monitoring concepts," in Medical Devices and Biosensors, 2008. ISSSMDBS 2008. 5th International Summer School and Symposium on, 2008, pp. 94-98.

[3] S. Saadaoui; and L. Wolf, "Architecture concerpt of a wireless body area sensor network for health monitoring of elderly people," in 4th IEEE Consumer Communications and Networking Conference, 2007, pp. 722-726.

[4] L. B and Y. Guangzhong . , "Body sensor networks:Research challenges and opportunities " in Proc of the IET Seminar on Antennas and Propagation for Body-Centfic Wireless Communications 2007, pp. 26-32.

[5] O. Aziz, B. Lo, A. Darzi, and G. Yang, Body sensor network: Springer, 2011.

[6] J. Y. K. Mehmet R. Yuce, Wireless body area networks : technology, implementation, and applications Imprint Singapore: Pan Stanford Pub, 2012.

[7] A. Boulis, D. Smith, D. Miniutti, L. Libman, and Y. Tselishchev, "Challenges in Body Area Networks for Healthcare," IEEE Communication Magazine, vol. 50, pp. 100-106, 2012.

[8] G. Fang;, E. Dutkiewicz, M. A. Huq, R. Vesilo, and Yihuai Yang, "Medical Body Area Networks: Opportunities, challenges and practices," in Communications and Information Technologies (ISCIT), 2011 11th International Symposium on 2011, pp. 562-567.

[9] I. S. ASSOCIATION, "IEEE Standard for Local and metropolitan area networks," in Part 15.6: Wireless Body Area Networks, ed. New York,USA: IEEE Computer Society, 2012.

[10] H. C. Keong and M. R. Yuce, "Analysis of a Multi-Access Scheme and Asynchronous Transmit-Only UWB for Wireless Body Area Networks," presented at the IEEE Engineering in Medical and Bioloy Conference, 2009.

[11] S. Ullah, M. Chen, and K. S. Kwak, "Throughput and delay analysis of IEEE 802.15.6-based CSMA/CA protocol," J Med Syst, vol. 36, pp. 3875-91, Dec 2012.

[12] K.-P. Shih, Y.-D. Chen, and C.-C. Chang, "A Physical/Virtual Carrier-Sense-Based Power Control MAC Protocol for Collision Avoidance in Wireless Ad Hoc Networks," IEEE Transactions on Parallel \& Distributed Systems, vol. 22, pp. 193-207, 2011.

[13] Pangun Park, Carlo Fischione, and a. K. H. Johansson, "Adaptive IEEE 802.15.4 Medium Access Control Protocol for Control and Monitoring Applications," in Wireless
Networking Based Control, ed New York ; London Springer, 2011, pp. 271-300.

[14] A. M. Asadi, V., " A Survey on Opportunistic Scheduling in Wireless Communications," Communications Surveys \& Tutorials, IEEE, vol. 15, pp. 1671-1688, 2013.

[15] S. G. Mario eagalj*, Imad Aad* and Jean-Pierre Hubaux, "On selfish behavior CSMA/CA," presented at the 24th Annual Joint Conference of the IEEE Computer and Communications Societies. , 2005.

[16] A. Boulis; and Y. Tselishchev. ContentionPolling:A Study in Body Area Network [Online].

[17] J. Tian, "Game-theory Model based on Carrier Sense Multiple Access Protocol in Wireless Network," Journal of Networks, vol. 9, 2014.

[18] G. Bianchi, L. Fratta, and M. Oliveri, "Performance evaluation and enhancement of the CSMA/CA MAC protocol for 802.11 wireless LANs," in Personal, Indoor and Mobile Radio Communications, 1996. PIMRC'96, Seventh IEEE International Symposium on 1996, pp. 392-396.

[19] P. Pangun, C. Fischione, and K. H. Johansson, "Performance Analysis of GTS Allocation in Beacon Enabled IEEE 802.15.4," in Sensor, Mesh and Ad Hoc Communications and Networks, 6th Annual IEEE Communications Society Conference on, 2009, pp. 1-9.

[20] S. Ullah, M. Mohaisen, and M. A. Alnuem, "A Review of IEEE 802.15.6 MAC, PHY, and Security Specifications," International Journal of Distributed Sensor Networks, vol. 2013, pp. 1-12, 2013.

[21] Benoît Latré, Pieter De Mil, Ingrid Moerman, and B. D. a. P. Demeester, "Throughput and Delay Analysis of Unslotted IEEE 802.15.4," JOURNAL OF NETWORKS, vol. 1, 2006.

[22] W. Hu, Q. Chen, P. Corke, and D. O'Rourke, "An energy-efficient rate adaptive media access protocol (RA-MAC) for long-lived sensor networks," Sensors vol. 10, pp. 5548-5568, 2010.

[23] Z. Han, Game theory in wireless and communication networks : theory, models, and applications New York: Cambridge University Press, 2011.

[24] T. Cui;, L. Chen;, and S. H, "A Game-Theoretic Framework for Medium Access Control," IEEE Journal on selected areas in communications vol. 26, pp. 1116-1126, 2008.

[25] C. Lijun, S. H. Low, and J. C. Doyle, "Contention control: A game-theoretic approach," in Decision and Control, 2007 46th IEEE Conference on, 2007, pp. 3428-3434.

[26] J. Zhou, S. Su, A. Guo, and W. Chen, "Abnormalities Detection of IMU based on PCA in motion monitoring," Applied Mechanics and Materials, vol. 224, pp. 533-538, 2012. 
Jing Zhou is currently pursuing the $\mathrm{PhD}$ degree in the Faculty of Engineering and IT, University of Technology, Sydney (UTS). She is a lecture in the School of Electronics and Information Engineering, Tongji University, China. Her main research interests include body area network, wireless communication, wearable monitoring system, tolerant control, and computational intelligence.

Aihuang Guo received his PhD in Xi'an Jiaotong Univerisity, China. He is a professor in School of Electronics and Information, Tongji University, China. His main research is in the area of the theory, technology and networks of broadband wireless communication.
Hung T. Nguyen received his BE degree with First Class Honors and $\mathrm{PhD}$ degree from the University of Newcastle in Australia. He has been involved with research in the areas of biomedical engineering, advanced control and artificial intelligence. He has developed several biomedical devices and systems for diabetes, disability, cardiovascular diseases and breast cancer.

Steven W. Su received his $\mathrm{PhD}$ degree in Australia National University. He is currently a Senior Lecturer in the Faculty of Engineering and IT, University of Technology, Sydney (UTS). His main research interests include biomedical system modeling and control, biomedical signal processing, wearable monitoring system, robust and adaptive control, fault tolerant control, intelligent control, and computational intelligence. 